\title{
The State of the Art in the Development of a Versatile Argumentation System based on the Logic of Multiple-valued Argumentation
}

\author{
Satoru Tannai ${ }^{1}$, Shogo Ohta $^{1}$, Takeshi Hagiwara ${ }^{2}$, Hajime Sawamura ${ }^{2}$ and Jacques Riche ${ }^{3}$ \\ ${ }^{1}$ Graduate School of Science and Technology, Niigata University, Niigata, Japan \\ ${ }^{2}$ Institute of Science and Technology, Niigata University, Niigata, Japan \\ ${ }^{3}$ Department of Computer Science, Katholieke Universiteit Leuven, Leuven, Belgium \\ \{tannai,s-ohta\}@cs.ie.niigata-u.ac.jp, \{hagiwara, sawamura\}@ie.niigata-u.ac.jp,riche@cs.kuleuven.ac.be
}

\begin{abstract}
Keywords: Multiple-valued Argumentation, Neural Net Argumentation, Eastern argumentation, Syncretic Argumentation, Argument Mining, Argument Animation.

Abstract: $\quad$ This paper reports on the state of the art in the development of a versatile argumentation system in which various auxiliary features for argumentation are incorporated. Such an argumentation system was built on our underlying argumentation system, LMA: the Logic of Multiple-valued Argumentation, aiming at promoting our understanding of argumentation processes. We in particular present some very new and unique aspects of computational argumentation: the syncretic argumentation, the argument mining and frequent sub-argument discovery, the argument metamorphosis from symbol to animation via natural language, Eastern argumentation based on Indian logic, and the argumentation based on symbolism and connectionism. Such a hybridization of various features would broaden the scope of the applications of computational argumentation in various ways.
\end{abstract}

\section{INTRODUCTION}

Argumentation is a dialectical process of knowing things (inquiry) and justifying them (advocacy) in general. Significances of argumentation have been recently recognized, and logical models of it have emerged as a promising paradigm for modeling agent reasoning and communication in light of interaction with other agents and arguments. Among other things, it has such a nature that closely mirrors the way humans reason and hence provides us a general framework for inference and decision making in the presence of inconsistent, uncertain and incomplete information.

We have developed a computational argumentation framework that basically consists of EALP and LMA (Takahashi and Sawamura, 2004). EALP (Extended Annotated Logic Programming) is an expressive logic programming language we formalized for argumentation. The basic language constituents are literals associated with annotations as truth-values or epistemic states of agents. LMA is a Logic of Multiple-valued Argumentation constructed on top of EALP. It has three notions of negation to yield a momentum or driving force for argumentation. LMA is a generic logic of multiple-valued argumentation that allows us to specify various types of truth values depending on application domains, and to deal with uncertain arguments. Such a feature brings us the extensive applicability of LMA that is considered the most advantageous point in comparison to other approaches to argumentation (Rahwan and Simari, 2009).

This paper reports on the state of the art in the development of a versatile argumentation system in which various auxiliary features for argumentation are incorporated on top of EALP/LMA, aiming at promoting our understanding of argumentation processes. We in particular present some very new and unique aspects of computational argumentation: the syncretic argumentation, the argument mining and frequent sub-argument discovery, the argument metamorphosis from symbol to animation via natural language, Eastern argumentation based on Indian logic, and the argumentation based on symbolism and connectionism. Such a hybridization of various features would broaden the scope of the applications of computational argumentation in various ways.

The paper is organized as follows. In the next section, we briefly illustrate part of EALP and LMA. In Section 3, we present an overall picture of IAE (Integrated Argumentation Environment) for arguing 
agents on the Internet, augmented with various auxiliary features: the syncretic argumentation, the argument mining and frequent sub-argument discovery, the argument metamorphosis from symbol to animation via natural language, Eastern argumentation based on Indian logic, and the argumentation based on symbolism and connectionism. In the succeeding sections, we briefly describe those features one by one. The final section includes concluding remarks and future work.

\section{OUTLINE OF EALP AND LMA}

\subsection{Knowledge Representation and Argument in EALP and LMA}

EALP (Extended Annotated Logic Programming) is an extended version of the Annotated Logic Programming (ALP) (Schweimeier and Schroeder, 2005) by introducing the extra negations below. Suppose the followings are knowledge bases that agent $\mathrm{A}$ and $\mathrm{B}$ have for argumentation respectively.

AgentA :

climb(satoru, mountain) $: 0.9 \leftarrow$ reflesh(satoru) $: 0.8$.

reflesh (satoru) $: 0.9 \leftarrow$ true.

AgentB :

$\sim \operatorname{climb}$ (satoru, mountain) : 0.6

$\leftarrow$ not $\sim$ get_tired_from(satoru, walking) : 0.8.

Real values are annotations for literals. They represent degrees of agents' beliefs. For example, we could read climb(satoru,mountain) : 0.9 as 'Satoru surely climb mountain.' The symbol $\sim$ stands for explicit negation (Takahashi and Sawamura, 2004). We could read $\sim$ climb(satoru, mountain) : 0.6 as 'Satoru possibly does't climb mountain.' The symbol not stands for default negation (Takahashi and Sawamura, 2004). We could read not $\sim$ get_tired_from(satoru,walking): 0.8. as 'There is no evidence for now to show that Satoru does't get tired from walking.'

An argument is a list of rules (knowledge). For example, the following Arg can be an argument, since the truth degree 0.9 is greater than 0.8 , or put it differently, the truthfood of reflesh(satoru): 0.9 includes that of reflesh(satoru) : 0.8 .

Arg $=[\operatorname{climb}($ satoru, mountain $): 0.9 \leftarrow$ reflesh $($ satoru $):$ 0.8., reflesh (satoru) : $0.9 \leftarrow$ true.]

The head of a rule is called a conclusion of the rule, and the annotated default literals in the body of the rule is called assumptions of the rule. We write concl(Arg) for the set of conclusions and assm(Arg) for the set of assumptions of the argument $\mathrm{Arg}$.
An argumentation is a finite nonempty sequence of moves move $_{i}=\left(\right.$ Player $_{i}$, Arg $\left._{i}\right)(i>0)$ that meets the following conditions.

1. Player $_{i}=P$ iff $i$ is odd; and Player $_{i}=O$ iff $i$ is even.

2. If Player $_{i}=$ Player $_{j}=P$ and $i \neq j$, then $\operatorname{Arg}_{i} \neq$ $\operatorname{Arg}_{j}$.

3. If $i \geq 2$, then $A r g_{i}$ defeats $A r g_{i-1}$.

\subsection{Judgement of Winning Dialogues}

In LMA, whether an agenda of argumentation is justified or not is judged based on the grounded semantics in Dungean semantics (Dung, 1995). In particular, the results of argumentation are displayed as dialogue trees based on the dialectical proof theory that corresponds to the grounded semantics (Schweimeier and Schroeder, 2005). A dialogue tree is a tree in which the node of the tree represents an argument and the branch of the tree represents an attack relation (Takahashi and Sawamura, 2004). A winning dialogue tree is a kind of dialogue tree in which every path in it is ended by the proponent's move $(P)$, and an agenda is justified if and only if at least one winning dialogue tree exists.

\section{A VERSATILE IAE}

\subsection{Integrated Argumentation Environment (IAE)}

From this section, we will propose a more versatile argumentation system in which various auxiliary features for argumentation are incorporated on top of LMA. For this, the following features are considered and realized to support or extend the past LMA argumentation (see Fig. 2 for the overall architecture of the integration).

(1) The hybrid argumentation based on symbolism and connectionism (Makiguchi and Sawamura, 2007) (Gotou et al., 2011)

We constructed a neural network that can compute the full Dungean semantics such as the stable, preferred, complete extension in addition to the grounded extension (Dung, 1995) which the original LMA only dealt with.

In Section 4, we briefly outline our neural network argumentation. 2005)

(2) The Eastern argumentation (Sawamura et al.,

We considered a significant specialization of LMA to Tetralemma $\mathcal{F} O \mathcal{U} \mathcal{R}=\{\perp, \mathbf{t}, \mathbf{f}, \top\}$ with an 


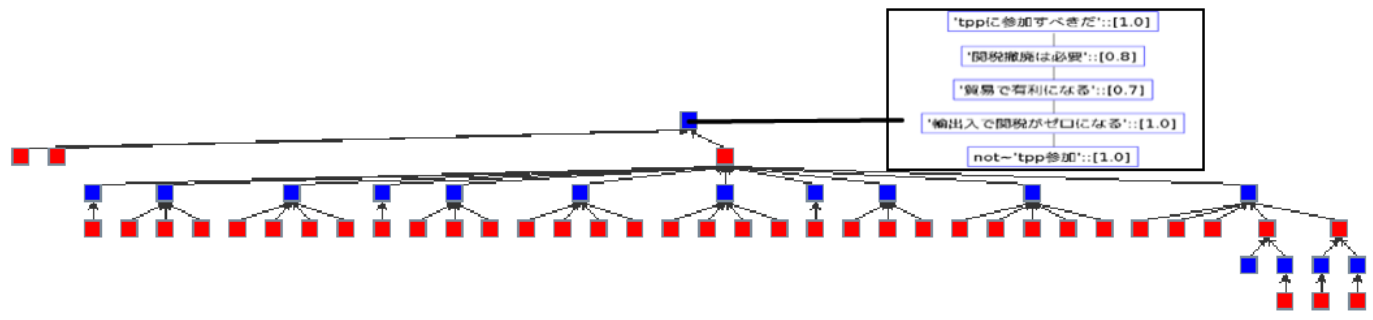

Figure 1: Argumentation graph of TPP, where the blue and red boxes denote arguments put forward, with the blue ones by the proponent side and the red ones by opponent side. The contents of the actual arguments pop up by clicking the nodes.

\section{Integrated Argumentation Environment (IAE)}

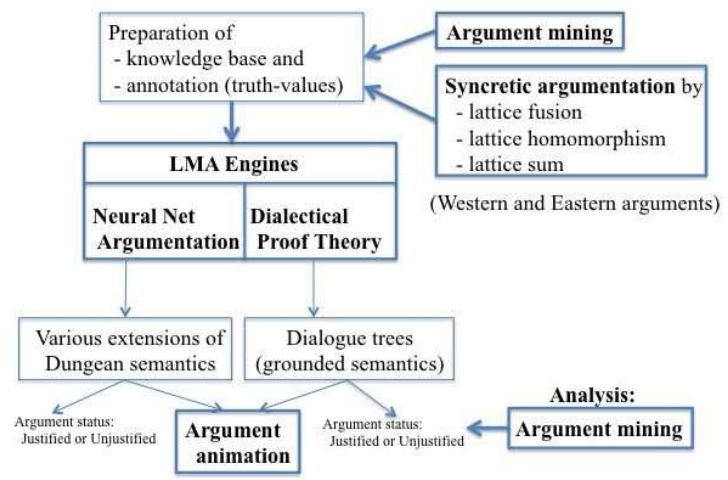

Figure 2: An overall picture of LMA-based argumentation system integrated with various auxiliary features.

Eastern mind, and confirm its expressiveness and applicability by applying it to arguments involving in Eastern thought and philosophy. We showed that LMA allows for a kind of pluralistic argumentation, or a fusion of Eastern and Western argumentation through cultural argument examples.

In Section 5, we, in particular, describe another kind of the Eastern argumentation and its semantics computation by the neural network argumentation mentioned (1) above.

(3) The syncretic argumentation based on LMA (Maruyama et al., 2012)

The syncretic argumentation framework is one that agents with different epistemology can engage in argumentation, taking into account the Golden Rule in the ethics of reciprocity and Confucius's Golden Rule. For example, back to the ancient, let us consider such a scene that Aristotle and Lao Tzu encounter, and argue about a proposition $p$. Perhaps, Aristotle might say $p$ is definitely true with his twovalued epistemology $\mathcal{T} \mathcal{W} O=\{\mathbf{f}, \mathbf{t}\}$, and Lao Tze might say $p$ may hold with truth degree $\perp$ with his four-valued epistemology $\mathcal{F}$ O $\mathcal{R} R=\{\perp, \mathbf{t}, \mathbf{f}, \top\}$. After all, they turn out to know that they can not communicate with each other. This is not just a matter of difference of knowledge, but difference of a way of recognizing things (epistemology), world-view, logic, and so on.

For the syncretic argumentation, we devised three kinds of lattice operations: homomorphism, fusion and sum that are to be described in Section 6.

(4) The frequent sub-argument mining and discovery (Seino et al., 2011)

Frequent sub-tree mining is useful since it enables agents to prepare or discover meaningful knowledge to be used for argumentation (Seino et al., 2011). For this, we employed the frequent subtree mining algorithm SLEUTH (Zaki, 2005) since it allows to mine embedded and induced subtrees which turn out to bring useful and meaningful substructures (subarguments) from argument databases to us. More in Section 7.

(5) Argument animation: the argument metamorphosis from symbol to animation via natural language (Narita and Sawamura, 2008)

Imaging of information is an effective way to assist us in easy and quick acquisition and understanding of information with personal sense organ. We demonstrated the way for imaging arguments among agents using the outcomes of LMA. It starts from a simple argument scenario with logical formulas produced by the dialectical proof theory of LMA. Then, it transforms to the natural language representation that is augmented with action and emotion of agents, and finally to a moving image of the resulting natural language scenario. Such an attempt might lead to a new approach to an electronic scenario writer and automatically creating moving images content. More in Section 8.

There has been no argumentation system so far like our versatile one in which those features concerned with argumentation are considered and integrated. In what follows, we will describe these features in more detail one by one.

\subsection{Case Studies}

We applied IAE to a somewhat realistic social issue: 'Should we join TPP:Trans-Pacific Partnership'. The 
result of the argumentation is displayed as a dialogue tree (see Fig. 1). This is not a winning dialogue tree. However, we may analyze our argument and build a winning dialogue tree through this dialogue tree, that is, by knowing weak points of our argument, supplementing more knowledge to agents, and so on. Later we will describe how to do it by our argument mining techniques.

\section{NEURAL NETWORK ARGUMENTATION}

The past IAE computed only the grounded semantics for argumentation (Takahashi and Sawamura, 2004). In order to allow to compute other Dungean semantics, we constructed a simple but versatile neural network for neural network argumentation, so that it can decide which argumentation semantics (admissible, stable, semi-stable, preferred, complete, and grounded semantics) a given set of arguments falls into, and compute argumentation semantics via checking (Gotou et al., 2011). Furthermore, we showed that such a neural network allows for the opposite direction from neural network computation to symbolic argumentation/dialogue (Gotou et al., 2011). In fact, We dealt with the question how various argumentation semantics can have dialectical proof theories, and described a possible answer to it by extracting or generating symbolic dialogues from the neural network computation under various argumentation semantics.

We will not describe the technical details for constructing a neural network for argumentation and its computing method in this paper (see (Goto et al., 2011) for them). Instead, we illustrate our basic ideas by using a simple argumentation graph and illustrating a neural network translated from it. We assume readers are familiar with the Dungean semantics such as admissible, stable, semi-stable, preferred, complete, and grounded semantics (Caminada, 2006)(Dung, 1995).

\subsection{Example of the Dungean Semantics Computed by the Neural Network in IAE}

Figure 3 shows a financial argument example computed by the neural network argumentation from the following arguments, where the first argument, for example, is a counterpart of the statement 'Spain is not Greece', asserting that Spain blames that Greece's financial situation is not sound as Spain believes that there is no evidence to show it's ill financial situation.

$A=[\sim$ greece $: 1.0 \leftarrow$ not $\sim$ spain $: 1.0]$

$B=[\sim$ greece $: 1.0 \leftarrow$ not $\sim$ portugal $: 1.0]$

$C=[\sim$ ireland $: 1.0 \leftarrow$ not $\sim$ greece $: 1.0]$

$D=[\sim$ ireland $: 1.0 \leftarrow$ not $\sim$ spain $: 1.0],[\sim$

portugal $: 1.0 \leftarrow$ not $\sim$ spain $: 1.0]$

$E=[\sim$ greekterritory $: 1.0 \leftarrow$ not $\sim$ ireland $: 1.0]$

$F=[\sim$ ireland $: 1.0 \leftarrow$ not $\sim \operatorname{spain}: 1.0 \&$ not $\sim$ portugal : 1.0$]$

$G=[\sim \operatorname{spain}: 1.0 \leftarrow$ not $\sim$ italy: 1.0$]$

$H=[\sim$ uganda $: 1.0 \leftarrow$ not $\sim \operatorname{spain}: 1.0]$

$I=[\sim \operatorname{spain}: 1.0 \leftarrow$ not $\sim$ uganda $: 1.0]$

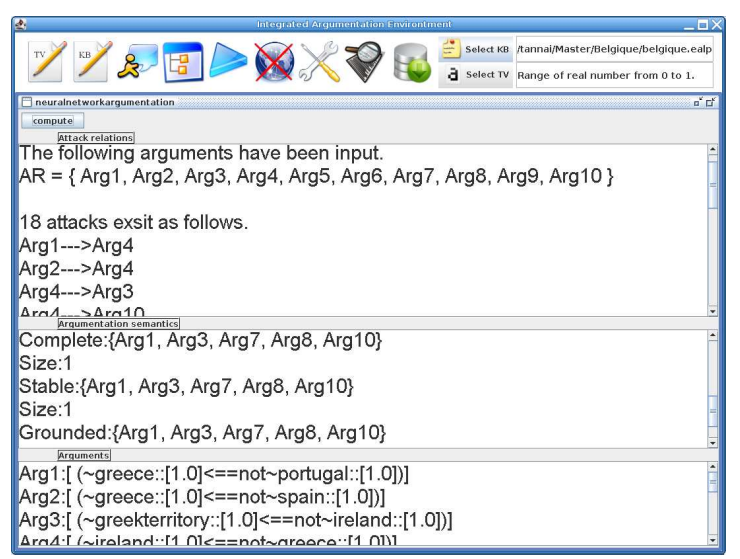

Figure 3: Financial argument by the neural network argumentation.

The argument engine constructed by the neural network has been shown to be useful since it allows to compute every Dungean semantics in a uniform manner. It is not simply a yet another alternative engine for argumentation. It plays an important role as the IAE argument engine.

\section{EASTERN ARGUMENTATION BASED ON INDIAN-LOGIC}

Truth is relative to culture (pluralism in philosophy) and can be brought through argumentation which is a true figure of logic (argumentation vs. logic). On the basis of this view or recognition, we have taken into account not only Western argumentation and logic but also Eastern argumentation and logic. In this section, we give an Eastern argumentation model and its adaptation to IAE. In particular, we adopt a typical way of thinking in the Indian logic, "five-membered syllogism (argument schema)" (Ganeri, 2001)(Mahalakshmi and Geetha, 2009), to our argumentation model. It is expected that it results in arguments with higher persuasion than western argumentation and logic only. 


\subsection{Five-membered Syllogism}

According to the ancient Indian logic, the proper formulation of the inference should have five parts. It is technically known as the five-membered inference or argument schema. According to Nyaya Sastra, an inferential proof is made up from the following members:

1. The statement

2. The cause or reason for the statement

3. The example (metaphor expression)

4. The application of that example

5. The conclusion

An example of the five-membered syllogism is as follows. (1) This hill has fire (statement), (2) Because, it has smoke (reason), (3) Since whatever has smoke has fire, e.g. an oven (example), (4) This hill has smoke, which is associated with fire (application), (5) Therefore, this hill has fire (conclusion)

\subsection{Eastern Argumentation Model}

Taking into account the five-membered syllogism, we construct an Eastern argumentation model in which attack relations are redefined so that the fivemembered syllogism may stand on predominance compared with other argument by considering 'persuasive power of statement'. In doing so, we introduce 'inductive clause' that can be included only in Eastern knowledge, and also specifies that the degree of predominance changes with examples used for Eastern arguments.

\subsubsection{An Inductive Clause (Indian Induction)}

The five-membered syllogism can be captured in terms of EALP as follows.

$A: \mu_{3} \leftarrow$ invariable $(B, A): \mu_{2} \& B: \mu_{1}$. invariable $(B, A): \mu_{2} \leftarrow$ ind (example $): \alpha$.

$B: \mu_{1} \leftarrow$ true.

Then the example of the five-membered syllogism mentioned above is expressed as follows.

has (hill, fire $): 1.0 \leftarrow$ invariable (smoke, fire $): 1.0$

\& has (hill, smoke) : 1.0.

invariable (smoke, fire) $: 1.0 \leftarrow$ ind (oven) $: \alpha$.

has (hill, smoke) $: 1.0 \leftarrow$ true.

where, the inductive clause, ind(oven) : $\alpha$ plays the roles of the third member of the five-membered syllogism: The example (metaphor expression) and the fourth member of the five-membered syllogism: The application of that example. Accordingly to the specific notations introduced, attack relations for arguments deserve to be changed so that eastern argu- ments may become advantageous by the relation between this $\alpha$ and annotations as truth values $\mu$ of other literals. The details are, however, omitted in this paper.

\subsubsection{Illustrative Example}

We illustrate a simple example of an Eastern argument. Suppose we have the following argumentation framework:

$A F_{1}=<\{A, B, C, D, E, F, G, H, I, J\},\{(A, E),(B, C)$, $(C, A),(C, D),(D, C),(E, A)\}>$.

Then we have the following extensions.

- Complete extension: $\{B, D, F, G, H, I, J\}$,

$\{A, B, D, F, G, H, I, J\},\{B, D, E, F, G, H, I, J\}$

- Stable extension : $\{A, B, D, F, G, H, I, J\}$,

$\{B, D, E, F, G, H, I, J\}$

- Grounded extension: $\{B, D, F, G, H, I, J\}$.

However, if we have the following framework in which the attack relation $(E, A)$ in $A F_{1}$ is blocked by the Eastern attack relation,

$A F_{2}=<\{A, B, C, D, E, F, G, H, I, J\},\{(A, E),(B, C)$, $(C, A),(C, D),(D, C)\}>$

then, we turn to have the following extensions.

- Complete extension: $\{A, B, D, F, G, H, I, J\}$

- Stable extension: $\{A, B, D, F, G, H, I, J\}$

- Grounded extension: $\{A, B, D, F, G, H, I, J\}$.

These computations have been done by the neural network argumentation of IAE.

\section{SYNCRETIC ARGUMENTATION}

We considered a novel approach to argumentation, called syncretic argumentation that allows agents to participate in argumentation even if they have knowledge bases with their own annotations as truth-values that reflect agents epistemic states of propositions. For the syncretic argumentation,we devised three methods: the lattice homomorphism, the fusion of latices, and the sum of lattices.

\subsection{The Lattice Homomorphism}

The mathematical structure of annotations in LMA is a complete lattice and the homomorphism is a mathematical apparatus convenient to syncretize the difference of epistemic states of propositions. It guarantees agents to retain agents' epistemic structure when embedding one lattice to the other. We also considered the bi-directional homomorphism on lattices since it allows for a fair, unbiased and pluralistic argumentation, prohibiting unilateral one. 
Let us consider two typical lattices: the twovalued complete lattice $\mathcal{T} \mathcal{W} O$ and the four-valued one F O I R . The former is typical in the West, and the latter in the early philosophical literature and text of Buddhism (Sawamura and Mares, 2004). $\mathcal{T} \mathcal{W} O=<\{f, t\}, \vee, \wedge, \leq>$, where $f \leq t$, and $\mathcal{F} O \mathcal{R} R=<\{\perp, \mathbf{t}, \mathbf{f}, \top\}, \vee, \wedge, \leq>$, where $\forall x, y \in$ $\{\perp, \mathbf{t}, \mathbf{f}, \top\} x \leq y \Leftrightarrow x=y \vee x=\perp \vee y=\top$. Note that we associate the suffix with annotations to avoid ambiguity of the same annotation names, that is, $t_{2}$ represents the annotation $t$ in $\mathcal{T} \mathcal{W} O$ and $t_{4}$ represents the annotation $t$ in $\mathcal{F} O \mathcal{U} \mathcal{R}$, for example.

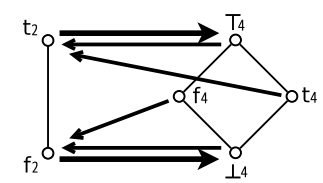

Figure 4: Lattice homomorphism.

\subsection{The Fusion of Lattices}

It is induced through the lattice product, and can be considered as providing a natural way to syncretize the difference of epistemic states of propositions. The fusion of lattices is formally defined(Maruyama et al., 2012).

Our approach to syncretic argumentation has such advantages as majority principle, order preserving and commutativity. Fig. 5 shows the fusion of $\mathcal{T} \mathcal{W} O$ and $\mathcal{F} O \mathcal{U R}$.

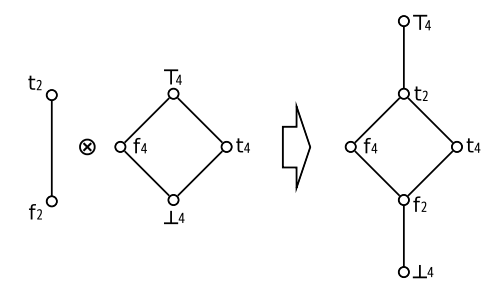

Figure 5: Fusion of $\mathcal{T} \mathcal{W} O$ and $\mathcal{F} O \mathcal{U} R$.

\subsection{The Horizontal Sum of Lattices}

The following is one of the lattice sum operations, called the horizontal sum of lattices (Davey and Priestley, 2002).

The syncretism by the lattice sum keeps the lattice structure of each agent intact, only identifying the minimal and maximal elements of it with those of the common ground. This better serves to act as a brake on excessive conflicts among agents. It is so-called a peaceful syncretic argumentation. Fig. 6 is the horizontal sum of $\mathcal{T} \mathcal{W} O$ and $\mathcal{F} O \mathcal{U} \mathcal{R}$.

Using either of these methods for the syncretic argumentation, agents get to communicate and enter their argumentation process.

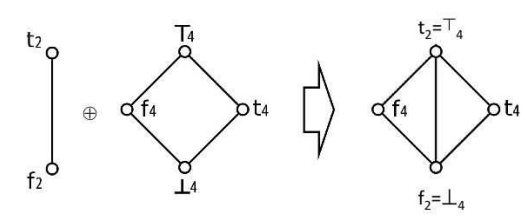

Figure 6: Horizontal sum of $\mathcal{T} \mathcal{W} O$ and $\mathcal{F} O \mathcal{U} \mathcal{R}$.

\section{ARGUMENT MINING}

The roles of argument mining for IAE development are twofold. As illustrated in Fig2, it helps the knowledge preparation in the process of IAE as agents want to collect their own knowledge for argumentation that are hopefully more efficient and persuasive, and furthermore generally approved. The other is that when an argument has not been justified, agents might want to analyze and turn it to a justified one with help of mined arguments that are resistant enough for counterarguments. Fortunately we now live in the world full of information, in particular, information of argument form, which are ceaselessly produced every day of our lives not only in the traditional media but also in the social WEB like Twitter, Facebook, SNS and many others. Such a 'Big Data' has become so valuable that it is deemed a new class of economic asset, such as oil.

\subsection{Argument Data Preparation}

A (reasonable) argument is a tree whose top leaves are premises and assumptions, and root is a conclusion, just like a (formal) proof. The argument data for argument mining consists of such argument trees. Contemporary argument data source largely comes from the social Web such as SNS, Blog, Twitter, etc., and are written in natural languages. So, we employed Araucaria (Reed and Rowe, 2004)(Araucaria, 2008) which is an argument mapping tool for analyzing, diagramming and representing arguments, for the data preparation of our argument mining. One of the unique features in Araucaria is that analyzed arguments can be saved in a portable format called AML (Argument Markup Language) that is a special XML stipulated in the document type definition, argument.dtd, particularly designed for arguments.

\subsection{Frequent Subtree Mining}

The frequent subtree mining is in general one of NP problems. The number of induced or embedded subtrees for a labeled tree $T$ grows exponentially with the size of $T$. Various frequent subtree mining algo- 
rithms, therefore, have been proposed so far, corresponding with targeted tree database types and substructure types to be discovered. In this paper, we employed SLEUTH algorithm (Zaki, 2005) for our purpose since the input of this algorithm is a set of unordered trees, the output is embedded subtrees, and it has the option for induced subtrees as well.

In our former paper (Seino et al., 2011), we showed that the so-called proof by contradiction (PBC, reductio ad absurdum) is mined from a formal proof data base. The assumption and conclusion of $\mathrm{PBC}$ are in the ancestor relationship.

In addition, we applied the frequent argument mining to the knowledge discovery for unjustified arguments. Fig. 7 is a screenshot of IAE that displays mined knowledge on the upper pane and selected knowledge from among it by a user on the lower pane. Fig 8 is a screenshot of an argument graph on an argument at the top node in which it is not justified, and Fig 9 is a screenshot of an argument graph with some arguments added by the argument mining, where the argument turns out to be justified.

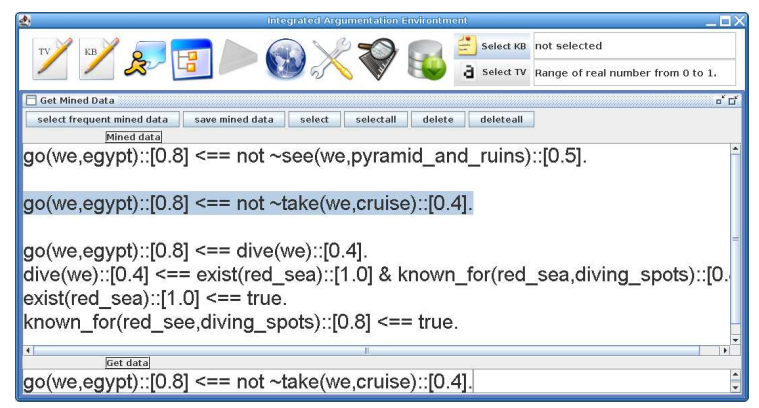

Figure 7: Knowledge extraction by the frequent argument mining.

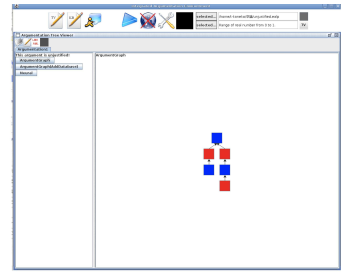

Figure 8: Argument graph without justification.

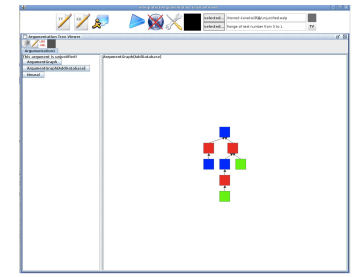

Figure 9: Argument graph with justification by argument mining.

\section{ARGUMENT ANIMATION}

IAE can be viewed as a scenario writer since it can produce an argumentative dialogue done among agents. We have been further promoting this observation to argument animation (Narita and Sawamura,
2008), (DMD, 2008). The way to this consists of three phases.

1. Producing a dialogue process in logical formulas by IAE

2. Translating it to a natural language dialogue by a simple language translation

3. Generating an animation script from it with the help of humans.

For example, the following unscientific and empirical but persuasive phrase in EALP

weather_is_good $: 0.7 \leftarrow$ sky_is_dyed_red_by_sunset $: 0.8$

is almost directly translated to

If the sky is considerably dyed red by sunset, the weather is surely good

in the preparation of a corresponding list of annotations and adverbs or adjectives. The third phrase can be semi-automatically accomplished by using an animation generation software that usually has a script language proper to animation generation. Figure 10 shows one shot of an argument animation of an argument on a trip to Egypt.

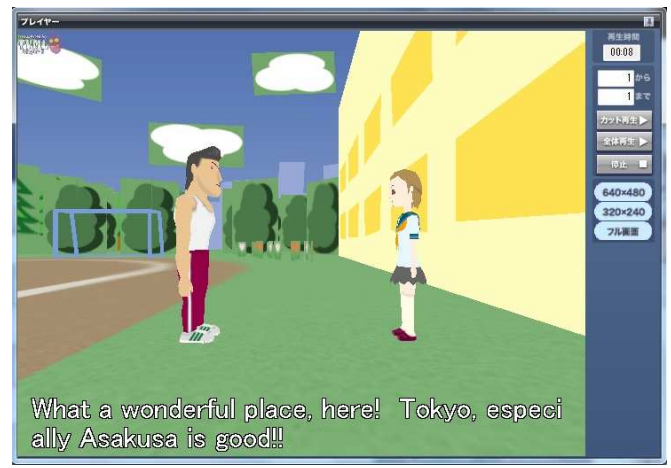

Figure 10: A slot of an argument animation generated by DMD.

\section{CONCLUSIONS}

In this paper, we have reported on the state of the art in the development of a versatile argumentation system based on the logic of multiple-valued argumentation. The distinctive features of it are two-fold. One is that its underlying theory is the logic of multiple-valued argumentation with which uncertain arguments can be dealt with under uncertain knowledge for argumentation. Such an argumentation model has been and still is rare in the community of argumentation. The other is that our argument system is versatile in the sense that it is equipped with the following unexemplified apparatuses:

- Hybrid argumentation based on symbolism and connectionism 
- Eastern argumentation based on Indian logic

- Syncretic argumentation

- Argument mining based on the frequent subargument mining and argument discovery

- Argument animation

These are aspects of argumentation in our argument activities we meet in our daily life as well except the first. Now we could say we have developed a very rich argument system, compared with other works (Rahwan and Simari, 2009).

We will further develop the system to incorporate the shift from argumentation to dialogue and the learning triggered by argumentation.

\section{REFERENCES}

Araucaria (2008). Araucaria version3.1-USER MANUAL-.

Caminada, M. (2006). Semi-stable semantics. In Dunne, P. E. and Bench-Capon, T. J. M., editors, Computational Models of Argument: Proceedings of COMMA 2006, volume 144 of Frontiers in Artificial Intelligence and Applications, pages 121-130. IOS Press.

Davey, B. A. and Priestley, H. A. (2002). Introduction to Lattices and Order. Cambridge.

DMD (2008). Digital Movie Director. http://www. movie-school.org/.

Dung, P. (1995). On the acceptability of arguments and its fundamental role in nonmonotonic reasoning, logics programming and n-person games. Artificial Intelligence, 77:321-357.

Ganeri, J. (2001). Indian Logic. Curzon Press.

Goto, Y., Hagiwara, T., and Sawamura, H. (2011). Neural networks computing the dungean semantics of argumentation. In NCTA 2011 - Proceedings of the International Conference on Neural Computation Theory and Applications, pages 5-14.

Gotou, Y., Hagiwara, T., and Sawamura, H. (2011). Extracting argumentative dialogues from the neural network that computes the dungean argumentation semantics. In 7th International Workshop on NeuralSymbolic Learning and Reasoning(NESY2011). IEEE.

Mahalakshmi, G. S. and Geetha, T. V. (2009). An indian logic-based argument representation formalism for knowledge-sharing. Logic Journal of the IGPL, 17(1):55-76.

Makiguchi, W. and Sawamura, H. (2007). A hybrid argumentation of symbolic and neural net argumentation (part i, part ii). In Rahwan, I., Parsons, S., and Reed, C., editors, ArgMAS, volume 4946 of Lecture Notes in Computer Science, pages 197-215/216-233. Springer.

Maruyama, Y., Hasegawa, T., Hagiwara, T., and Sawamura, H. (2012). Syncretic argumentation for multi-agents by lattice homomorphism, fusion and sum. In Argumentation in Multi-Agent Systems, 8th International
Workshop, ArgMAS 2011, Revised Selected and Invited Papers, volume 7543 of Lecture Notes in Computer Science, pages 46-65. Springer.

Narita, T. and Sawamura, H. (2008). An attempt to argument metamorphosis from symbols to moving images. In Proceedings of 2008 Joint Agent Symposium (JAWS2008).

Rahwan, I. and Simari, G. R. E. (2009). Argumentation in Artificial Intelligence. Springer.

Reed, C. and Rowe, G. (2004). Araucaria: Software for argument analysis, diagramming and representation. International Journal on Artificial Intelligence Tools, 13(4):983-.

Sawamura, H. and Mares, E. (2004). How agents should exploit tetralemma with an eastern mind in argumentation. In Mike Barley and Nik Kasabov (eds.): Intelligent Agents and Multi-Agent Systems VII, Lecture Notes in Artificial Intelligence, Springer, volume 3371 , pages $259-278$.

Sawamura, H., Takahashi, T., and Matsunaga, K. (2005). An eastern specialization of logic of multiple-valued argumentation to tetralemma originated in india. In Prasad, B., editor, IICAI, pages 1274-1291. IICAI.

Schweimeier, R. and Schroeder, M. (2005). A parameterised hierarchy of argumentation semantics for extended logic programming and its application to the well-founded semantics. TPLP, 5(1-2):207-242.

Seino, Y., Sawamura, H., and Hagiwara, T. (2011). Towards argument mining. In Proceedings of 2011 International Conference on Data Engineering and Internet Technology (DEIT 2011), pages 27-34. IEEE.

Takahashi, T. and Sawamura, H. (2004). A logic of multiple-valued argumentation. In Proceedings of the third international joint conference on Autonomous Agents and Multi Agent Systems (AAMAS'2004), pages $800-807$. ACM.

Zaki, M. J. (2005). Efficiently mining frequent embedded unordered trees. Fundam. Inform., 66(1-2):33-52. 